\title{
Antioxidant enzymes induction in pea plants under high irradiance
}

\author{
J.A. HERNÁNDEZ ${ }^{1 *}$, C. ESCOBAR ${ }^{* *}$, G. CREISSEN ${ }^{* * *}$, P. M. MULLINEAUX ${ }^{* * * *}$
}

Dept. de Mejora y Fisiología Vegetal. Centro de Edafología y Biología Aplicada del Segura, CSIC, Apartado 164, E-30100 Espinardo-Murcia, Spain ${ }^{*}$

Facultad de Ciencias del Medio Ambiente, Universidad de Castilla-La Mancha. Campus de la Fábrica de Armas, E-45071 Toledo, Spain ${ }^{* *}$

Dept. Disease and Stress Biology, John Innes Centre, Norwich Research Park, Colney, Norwich NR4 7UH, UK ${ }^{* * *}$

Univ Essex, Dept Biol Sci, Wivenhoe Park , Colchester, Essex CO4 3SQ England $^{* * * *}$

Abbreviations: AOS - Activated oxygen species; APX - ascorbate peroxidase; ASC-GSH cycle - ascorbate-glutathione cycle; CAT - catalase; CuZn-SOD - copper,zinccontaining superoxide dismutase; DHAR - dehydroascorbate reductase; HI - high irradiance; GR - glutathione reductase; GPX - glutathione peroxidase; $\mathrm{H}_{2} \mathrm{O}_{2}$ - hydrogen peroxide; MDHAR - monodehydroascorbate reductase; SOD - superoxide dismutase; $\mathrm{O}_{2}{ }^{-}$ - superoxide radicals; PHGPX - phospholipid hydroperoxide glutathione peroxidase

${ }^{1}$ Corresponding author. Tel.: +34-968-396-200; fax: +34-968-396-213

E-mail: jahernan@cebas.csic.es 


\begin{abstract}
Exposure of pea plants to high irradiance (HI) for 60 min caused a reversible photo inhibition of photosynthesis as shown by changes in variable to maximum fluorescence ratio $\left(F_{v} / F_{m}\right)$. A significant decline in $F_{m}$ was observed in leaves from both pea cultivars subjected to HI, the decrease being higher in JI281 than in JI399 plants. The values recovered during the post-stress period in both cultivars. In both cultivars, under HI increased $\mathrm{F}_{0}$, but in cultivar JI399 initial values were recovered during the post-stress period.
\end{abstract}

The expression of antioxidant enzyme genes was higher in JI399 than in JI281, both in control and stressed plants. In JI281, after 60 min of HI, an induction of the transcripts of CAT, chlMDHAR, cytAPX and cytCu,Zn-SOD was observed, whereas there were a slight increase in PHGPX, stAPX and chlCu,Zn-SOD mRNAs. After $24 \mathrm{~h}$ of the recovery period, the induction of some transcripts was not maintained (CAT, cytAPX and cytCu,Zn-SOD), whereas the induction of others was maintained (PHGPX and chlCu,ZnSOD) or even increased (cytGR, stAPX and chlMDHAR).

In JI399, CAT and cytAPX were increased strongly after $60 \mathrm{~min}$ of $\mathrm{HI}$, and slight increases were observed in cytGR, chlGR and chlMDHAR. In the poststress period the expression of stAPX, cytGR and chIMDHAR was even slightly higher than after $60 \mathrm{~min}$ of HI, however, expression of CAT, cytAPX, cytCu,ZnSOD, chlCu,ZnSOD and chlGR was decreased.

Additional keywords: antioxidants; gene expression; photoxidative stress; Pisum sativum

\title{
Introduction
}

High irradiance (HI) exposure is one of the most common causes of oxidative stress in plants (Dat et al. 2000). Under excess of irradiation, enzymatic processes for $\mathrm{CO}_{2}$ fixation become rate-limiting and, as a result, photosynthesis produces more NADPH and ATP than necessary. This accumulation of redox and energy equivalents will decrease the plastoquinone pool and/or inhibit the water-splitting complex, inevitably leading to PS 2 inactivation, the so-called photoinhibition (Anderson et al. 1997; Karpinski et al. 1997, 1999). 
There are reports on the changes in activity and expression of antioxidant enzymes in response to HI stress (Gillham and Dodge 1987; Foyer et al. 1989; Karpinski et al. 1997; Yoshimura et al., 2000; Hernández et al. 2004) but the results vary according to plant materials and treatment conditions employed.

The protection of the plants against AOS produced in excess during adverse environmental conditions is achieved by means of different strategies and, in particular, by partial suppression of its production and scavenging of the AOS already produced (Murgia et al. 2004). During HI stress, free radicals are formed in PS 2, which are harmful to the photosynthetic apparatus (Aro et al. 1993). Partial suppression of AOS production in the chloroplasts during HI stress is achieved by degradation of the D1 protein of PS 2 leading to its inactivation (Anderson et al. 1997; Karpinski et al. 1997, 1999).

Plants contain a complex antioxidant system to detoxify AOS that includes carotenoids, ascorbate, glutathione, tocopherols, anthocyanin pigments and enzymes such as superoxide dismutase, catalase, glutathione peroxidase, peroxidases and the enzymes involved in the ascorbate-glutathione cycle (Foyer and Halliwell 1976): ascorbate peroxidase, dehydroascorbate reductase, monodehydroascorbate reductase and glutathione reductase.

In this work, we investigate the effect of EL on PS 2 efficiency in pea leaves, as well as on the levels of mRNAs encoding crucial enzymes of AOS metabolism.

\section{Materials and methods}

Plants, growth and treatments: Pisum sativum, cvs. JI281 and JI399, seedlings, individually planted in pots, were grown in a controlled-environment growth chamber (18-h photoperiod, irradiance of $200 \pm 25 \mu \mathrm{mol}$ (photon) $\mathrm{m}^{-2} \mathrm{~s}^{-1}$, temperature of $15 \pm 1.5^{\circ} \mathrm{C}$ and relative humidity of $75 \pm 5 \%$ ) (Ellis et al., 1992). JI281 is a semidomesticated land race of pea from Ethiopia whereas JI399 is a typical domesticated garden pea cultivar (Ellis et al. 1992).

Experiments were performed with 21-day-old pea plants exposed to HI exposure for up to $60 \mathrm{~min}\left(4000 \mu \mathrm{mol} \mathrm{m} \mathrm{m}^{-2} \mathrm{~s}^{-1}\right)$. To eliminate heat effects and to disperse light evenly, light was reflected by a mirror and directed through a frosted-glass filter filled 
with cold water. Control plants were exposed to $200 \pm 25 \mu \mathrm{mol} \mathrm{m} \mathrm{m}^{-2} \mathrm{~s}^{-1}$. After this period, some light-stressed plants were returned for $24 \mathrm{~h}$ to normal growth conditions (re-exposed to $200 \pm 25 \mu \mathrm{mol}$ of photons $\mathrm{m}^{-2} \mathrm{sec}^{-1}$ ) before sampling (poststress period). All leaves were frozen in liquid $\mathrm{N}_{2}$ and stored at $-70^{\circ} \mathrm{C}$ until use.

Measurement of chlorophyll fluorescence: The photosystem 2 efficiency parameter $\mathrm{F}_{\mathrm{v}} / \mathrm{F}_{\mathrm{m}}$ (the ratio of variable to maximal fluorescence reference), $\mathrm{F}_{0}$ (minimal chlorophyll fluorescence) and $F_{m}$ (maximal chlorophyll fluorescence) were measured using a portable Plant Efficiency Analyser (PEA) machine (Hansatech Instrument, King`s Lynn, Norfolk, UK), on 10 min dark-adapted leaves at 90 \% light saturation, according to the manufacturers' instructions.

Northen blotting: Total RNA from leaves was extracted as previously described (Creissen and Mullineaux, 1995). Poly $\mathrm{A}^{+}$-mRNA was purified by chromatography, using oligo $d(T)$ cellulose spin columns (Amersham Biosciences, UK) according to the manufacturer's instructions. Poly $\mathrm{A}^{+}$-mRNA (3-5 $\mu \mathrm{g}$ ) was denatured, separated electrophoretically and transferred onto a nitrocellulose membrane as described in Hernández et al (2000). The loading of an equivalent amount of Poly $\mathrm{A}^{+}$-mRNA for each time point was checked on gels stained with ethidium bromide and they showed equal intensities (data not shown). Northern blots were visualised by autoradiography after hybridisation with ${ }^{32} \mathrm{P}$-labelled DNA probes. Hybridisation was carried out in 0.3 M sodium phosphate buffer $\mathrm{pH}$ 7.2, containing $1 \mathrm{mM}$ EDTA, 7\% SDS and 1\% BSA, at $65^{\circ} \mathrm{C}$ (homologous probes) or $55^{\circ} \mathrm{C}$ (heterologous probes). Washing was with $0.1 \mathrm{X}$ SSC $\left(65^{\circ} \mathrm{C}\right)$ for homologous probes and with1 X SSC or 2 X SSC for heterologous probes. Northern blots were performed twice and representative blots are shown.

The specific probes used were: cytosolic and chloroplastic Cu,Zn-SOD from Nicotiana plumbaginifolia (Bowler et al. 1989; Van Camp et al. 1990), cytosolic cytGR (GOR 2, Stevens et al. 1997) and chloroplastic GR (GOR 1, Creissen and Mullineaux 1995), catalase from tobacco (Willekens et al. 1994), PHGPX (Mullineaux et al. 1998), chlMDAR from Arabidopsis (Genbank accession number T04550), cytosolic APX from pea (Santos et al. 1996) and stromal APX from spinach (Ishikawa et al. 1995). 
The filters were exposed to x-ray film and/or were visualised on a BAS 1000 Phosphoimager analyser (Fuji Photofilm Co., Kanagawa, Japan). Scanning values were calculated by BASIS software (Fuji Photofilm Co.) installed on the BAS 1000.

Statistics: Comparisons among means were made using Duncan's multiple range test, calculated at $\mathrm{P}<0.05$. Statistical procedures were carried out with the software package SPSS 11.0 for Windows.

\section{Results}

Exposure of 3-week-old pea plants at HI $\left(4000 \mu \mathrm{mol} \mathrm{m} \mathrm{m}^{-2} \mathrm{~s}^{-1}\right)$ for $60 \mathrm{~min}$ caused a significant photoinhibition in both cultivars as indicated by the decline in the PS 2 efficiency parameter $F_{v} / F_{m}$ (Fig. 1A). After $24 \mathrm{~h}$ at $200 \mu \mathrm{mol} \mathrm{m} \mathrm{s}^{-2}$, the efficiency of PS 2 had recovered as shown by the increase in $F_{v} / F_{m}$ (Fig. 1A) to see values of the control plants. In both cultivars, HI caused an increase in $\mathrm{F}_{0}$. In JI281, $\mathrm{F}_{0}$ partially recovered after $24 \mathrm{~h}$, but still was increased in relation to time zero. However, in JI399, $F_{0}$ values had reached control values after $24 \mathrm{~h}$ (Fig. 1B). A significant decline in $F_{m}$ was observed in leaves from both cultivars subjected to HI, the decrease being greater in JI281 than in JI399 plants. $F_{m}$ values recovered during the post-stress period in both cultivars.

The expression levels of antioxidant enzyme genes was higher in cultivar JI399 than in JI281, both in control and in stressed plants (Fig.1). In cultivar JI281, after $1 \mathrm{~h}$ of $\mathrm{HI}$, an induction of the transcripts of CAT, chlMDHAR, cytAPX and cytCu,Zn-SOD was observed, whereas there were a slight increases in PHGPX, stAPX and chlCu,Zn-SOD mRNAs. After $24 \mathrm{~h}$ of the recovery, some transcripts were not maintained (CAT, cytAPX and cytCuZn-SOD), whereas others were maintained (PHGPX and chlCu,ZnSOD) or even increased (cytGR, stAPX and chlMDHAR) (Fig.1, Table 1).

In cultivar JI399, CAT and cytAPX were increased after $1 \mathrm{~h}$ of $\mathrm{HI}$, and slight increases were observed in cytGR, chlGR and chlMDHAR (Fig. 1, Table 1). After $24 \mathrm{~h}$ recovery, the expression of CAT, stAPX, cytGR and chIMDHAR was slightly higher than after $60 \mathrm{~min}$ of HI (Fig. 1, Table 1). However, contents of cytAPX, cytCu,ZnSOD, chlCu,ZnSOD and chlGR transcripts were lower when compared to those exhibited in control plants (time zero) and after $1 \mathrm{~h}$ of HI stress (Fig. 1, Table 1). 


\section{Discussion}

Short-term exposure of pea plants to HI caused a significant photoinhibition, although $\mathrm{F}_{\mathrm{v}} / \mathrm{F}_{\mathrm{m}}$ values had recovered after $24 \mathrm{~h}$ of the post-stress period. A similar response in photosynthesis has been described in spinach and Arabidopsis plants as well as in detached pea leaves subjected to HI stress (Yoshimura et al. 2000; Karpinski et al. 1997; Hernández et al. 2004). The increase in $\mathrm{F}_{0}$ during HI has been described also in Arabidopsis plants as well as in detached pea leaves (Karpinski et al. 1997; Hernández et al. 2004). Elevated $\mathrm{F}_{0}$ (zero fluorescence) has been considered as reflecting thylakoid membrane disturbance and photoinhibitory damage, and decreases in $F_{v} / F_{m}$ and $F_{m}$ during $\mathrm{HI}$ indicate that photoinhibition of photosynthesis ocurred (Krause and Weiss 1991; Balachandran and Osmond 1994; Karpinski et al. 1997; Bertamini and Nedunchezhian 2004).

mRNAs from some genes encoding antioxidant enzymes accumulated in both cultivars during HI, although to different extents. At time zero, as well as in stressed and recovered plants, expression of all mRNAs was higher in cultivar JI399 than in JI281. This could contribute to a more efficient response to the AOS that may be generated in chloroplasts and cytosol during the $\mathrm{HI}$ and post-stress periods. However, after HI, the increases observed for chIMDHAR, cytCuZnSOD and cytAPX were higher in JI281 than in JI399 plants, which could be due to the lower AOS generation in JI399 compared to JI281 after HI, as shown previously in detached leaves from the same cultivars (Hernández et al. 2004).

The strong induction of CAT, cytAPX and cytCu,ZnSOD in JI281 indicates that $\mathrm{HI}$ could have increased $\mathrm{O}_{2}{ }^{--}$and $\mathrm{H}_{2} \mathrm{O}_{2}$ levels in the cytosol. In the same way, the induction of CAT and cytAPX in JI399 could indicate also an increased $\mathrm{H}_{2} \mathrm{O}_{2}$ generation in the cytosolic compartment. An increase in $\mathrm{H}_{2} \mathrm{O}_{2}$ has been observed also in detached leaves from these pea cultivars after 60 min of HI, being higher in JI281 than in JI399 (Hernández et al. 2004).

On the other hand, the higher expression of chlMDHAR, chlGR, chlPHGPX, stAPX and chlCu,Zn-SOD observed in JI399 under HI, could suggest a higher protection against AOS in chloroplasts from this pea cultivar. Probably, the greater alterations in $\mathrm{F}_{0}$ and $\mathrm{F}_{\mathrm{m}}$ observed in JI281 could be related with the lower expression of all antioxidant enzymes analysed. 
In detached pea leaves and in Arabidopsis plants, photoinhibition was correlated with an increase in $\mathrm{H}_{2} \mathrm{O}_{2}$ (Karpinski et al. 1997; Hernández et al. 2004). A similar response has been observed in spinach, where a transient increase in $\mathrm{H}_{2} \mathrm{O}_{2}$ levels was observed after $1 \mathrm{~h}$ of $\mathrm{HI}$, correlated with a drop in $\mathrm{F}_{\mathrm{v}} / \mathrm{F}_{\mathrm{m}}$ values (Yoshimura et al. 2000). So, the decrease in $F_{v} / F_{m}$ and $F_{m}$, as well as the increase in $F_{0}$, observed in pea plants subjected to HI could also be due to an increase in AOS in their chloroplasts. In bean plants, continuous irradiation induced premature senescence caused by enhanced production of AOS (Procházkova and Wilhelmová 2004).

The increase in cytGR transcripts observed in pea leaves after $24 \mathrm{~h}$ post-stress has been previously described in Arabidopsis plants and in detached pea leaves (Karpinski et al. 1997; Hernández et al. 2004), and a similar increase in chlMDHAR has been described also in detached pea leaves recovered from HI (Hernández et al. 2004). In wheat, DHAR activity declined during the early stages of seedling growth under a high-light regime and this implies that regeneration of ASC for scavenging of $\mathrm{H}_{2} \mathrm{O}_{2}$ is catalysed mostly by MDHAR and not by DHAR (Mishra et al. 1995). In the present study, we have not measured DHAR activity or expression, but the induction of chlMDHAR and the slight decrease in chlGR, in both pea cultivars, suggest that in chloroplasts, ASC seems to be regenerated mostly via MDHAR. Conversely, the increase in $c y t G R$ in recovered plants indicates that ASC could be regenerated mainly via GSH in the cytosolic compartment.

It has been suggested that in the induction of transcripts encoding for antioxidant enzymes under $\mathrm{HI}$, both $\mathrm{H}_{2} \mathrm{O}_{2}$ and the redox status of ascorbate could be involved (Hernández et al., 2004). However, in Arabidopsis plants, a role for the redox state of glutathione and the plastoquinone pool also has been proposed (Karpinski et al. 1997). Therefore, in the present study, and although no measurements of $\mathrm{H}_{2} \mathrm{O}_{2}$, ascorbate or glutathione levels have been carried out, the induction of transcripts encoding for antioxidant enzymes in response to $\mathrm{HI}$ could also have been mediated by AOS or by the alteration in the redox state of ascorbate and/or glutathione.

The data obtained about the expression of genes encoding antioxidant enzymes suggest that HI could alter AOS levels, both in the chloroplasts and cytosol of pea leaves. The induction of cytosolic antioxidant enzymes could provide an alternative protection when the chloroplastic defence systems are compromised under light stress conditions (Karpiski et al. 1997; Hernández et al. 2004). Support for this was reported by Yoshimura et al. (2000) who found that stress conditions, including HI, drought, 
salinity and methyl viologen treatments, which enhanced AOS in chloroplasts, also induces the cytosolic scavenging system.

In conclusion, results indicated that HI caused a reversible photoinhibition of photosynthesis in pea chloroplasts, and that changes in both $\mathrm{F}_{0}$ and $\mathrm{F}_{\mathrm{m}}$ contributed to the decrease in $\mathrm{F}_{\mathrm{v}} / \mathrm{F}_{\mathrm{m}}$, the changes being more important in JI281 than in JI399 leaves. Also, it seems that $\mathrm{HI}$ produces an increase in AOS that could regulate the accumulation of mRNAs encoding antioxidant enzymes. These data also suggest that pea cultivar JI399 seems to be relatively more tolerant than JI281 to HI, at least partly, due to its higher expression levels of antioxidant enzymes.

\section{References}

Anderson, J.M., Park, Y.I., Chow, W.S. Photoinactivation and photoprotection of photosystem II in nature. Physiol. Plant. 100: 214-223, 1997.

Aro, E.M., McCaffery, S., Anderson, J.M. Photoinhibition and D1 protein degradation in peas acclimated to different growth irradiances. Plant Physiol. 103: 835-843, 1993.

Balachandran, S., Osmond, C.B. Susceptibility of tobacco leaves to photoinhibition following infection with two strains of tobacco mosaic virus under different light and nitrogen nutrition regimes. Plant Physiol. 104: 1051-1057, 1994.

Bertamini, M., Nedunchezhian, N. Photosynthesis responses for Vitis vinifera plants grown at different photon flux densities under field conditions. Biol. Plant. 48: 149-152, 2004

Bowler, C., Alliotte, T., De Loose, M., Van Montagu, M., Inzè, D. The induction of manganese superoxide dismutase in response to stress in Nicotiana plumbaginifolia. EMBO J. 8: 31-38, 1989 
Creissen, G.P., Mullineaux, P.M.. Cloning and characterisation of glutathione reductase cDNAs and identification of two genes encoding the tobacco enzyme. Planta 197: 422-425, 1995.

Dat, J., Vandenabeele, S., Vranová, E., Van Montagu, M., Inzè, D., and Van Breusegem, F.. Dual action of the active oxygen species during plant stress responses. Cell. Mol. Life Sci. 57: 779-795, 2000.

Ellis, T.H.N., Turner, L., Hellens, R.P., Lee, D., Harker, C.L., Enard, C., Domoney, C., Davies, D.R.. Linkage maps in pea. Genetics 130: 649-663, 1992.

Foyer, C.H., Halliwell, B. The presence of glutathione and glutathione reductase in chloroplasts: a proposed role in ascorbic acid metabolism. Planta 133: 21-25, 1976.

Foyer, C.H., Dujardyn, M., Lemoine, Y. Responses of photosynthesis and the xanthophyll and ascorbate cycles to changes in irradiances, photoinhibition and recovery. Plant Physiol. Biochem. 27: 751-760, 1989.

Gillham, D.J., Dodge, A.D. Chloroplasts superoxide and hydrogen peroxide scavenging systems from pea leaves: seasonal variations. Plant Sci. 50: 105-109, 1987.

Hernández, J.A., Jiménez, A., Mullineaux, P.M., Sevilla, F. Tolerance of pea (pisum sativum l.) to long-term salt stress is associated with induction of antioxidant defences. Plant Cell Environ. 23: 853-862, 2000.

Hernández, J.A., Escobar, C., Creissen, G., Mullineaux, P.M. Role of hydrogen peroxide and the redox state of ascorbate in the induction of antioxidant enzymes in pea leaves under excess light stress. Function. Plant Biol. 31: 359368, 2004

Ishikawa, T., Sakai, K., Takeda, T., Shigeoka, S. Cloning and expression of cDNA encoding a new type of ascorbate peroxidase from spinach. FEBS Letters 367: 28-32, 1995. 
Karpinski, S., Escobar, C., Karpinska, B., Creissen, G., Mullineaux, P.M.. Photosynthetic electron transport regulates the expression of cytosolic ascorbate peroxidase genes in Arabidopsis during excess light stress. Plant Cell 9: 627640, 1997

Karpinski. S. Reynold, H., Karpinska, B., Wingsle, G., Mullineaux, P.M. Systemic signaling and acclimation in response to excess excitation energy in Arabidopsis. Science 284: 654-657, 1999.

Krause, G.H., Weiss, E.. Chlorophyll fluorescence and photosynthesis: The basics. Ann. Rev. Plant Physiol. Plant Mol. Biol. 42: 313-349, 1991.

Mishra, H.P., Fatma, T., Singhal, G.S. Development of antioxidative defense system of wheat seedlings in response to high light. Physiol. Plant. 95: 77-82, 1995.

Murgia,I., Tarantino, D., Vannini, C., Bracale, M., Carravieri, S., Soave, C. Arabidopsis thaliana plants overexpressing thylakoidal ascorbate peroxidase show increased resistance to paraquat-induced photooxidative stress and to nitric oxida-induced cell death. Plant J. 38: 940-953, 2004.

Mullineaux, P.M., Karpinski, S., Jiménez, A., Cleary, S., Robinson, C., Creissen, G. Plastid-targeted glutathione peroxidase provides evidence for an ascorbateindependent glutathione redox cicle in plant chloroplast. Plant J. 13: 375-379, 1998.

Procházková, D., Wilhelmová, N. Changes in antioxidant protection in bean cotyledons during natural and continuous irradiation-accelerated senescence. Biol. Plant., 48: 33-39, 2004.

Santos, M., Gousseau, H., Lister, C., Foyer, C., Creissen, G., Mullineaux, P.M. Cytosolic ascorbate peroxidase from Arabidopsis thaliana L. is encoded by a small multigene family. Planta 198: 64-69, 1996. 
Stevens, R., Creissen, G., Mullineaux, P.M. Cloning and characterization of a cytosolic glutathione reductase cDNA from pea (Pisum sativum L.) and its expression in response to stress. Plant Mol. Biol. 35: 641-654, 1997.

Van Camp, W., Bowler, C., Villarroel, R., Tsang, E.W.T., Van Montagu, M., Inzè, D.. Characterization of iron superoxide dismutase cDNAs from plants obtained by genetic complementation in Escherichia coli. Proc. Natl. Acad. Sci. USA 87: 9903-9907, 1990.

Willekens, H., Langebartels, C., Tiré, C., Van Montangu, M., Inzè, D., Van Camp, W. Differential expression of catalase genes in Nicotiana plumbaginifolia (L.). Proc. Natl. Acad. Sci. USA 91: 10450-10454, 1994.

Yoshimura, K., Yabuta, Y., Ishikawa, T., Shigeoka, S. Expression of spinach ascorbate peroxidase isoenzymes in response to oxidative stress. Plant Physiol. 123: 223233, 2000.

Table 1. Transcript levels relative to the time 0 of genes coding antioxidant enzymes from attached pea leaves exposed to EL. A representative Northern blot is shown. 
${ }^{a}$ Values were obtained after the scanning of RNA gel blot hybridization with cDNA probes. Value at time 0 is set to 1.

JI-281

JI-399

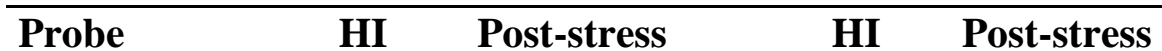

\begin{tabular}{lllll}
\hline chlMDHAR & 2.04 & 3.33 & 1.4 & 1.64 \\
GOR 2 & 1.35 & 2.22 & 1.21 & 1.50 \\
GOR 1 & 0.96 & 0.81 & 1.2 & 0.7 \\
CAT1 & 2 & 1.3 & 2.24 & 1.15 \\
PHGPX & 1.71 & 1.93 & 1.04 & 0.8 \\
cytAPX & 4.16 & 1.4 & 2.03 & 0.73 \\
stAPX & 1.4 & 3.15 & 0.5 & 1.4 \\
chlCuZnSOD & 1.53 & 1.33 & 0.97 & 0.60 \\
cytCuZnSOD & 3.36 & 1.95 & 0.8 & 0.62 \\
\hline
\end{tabular}



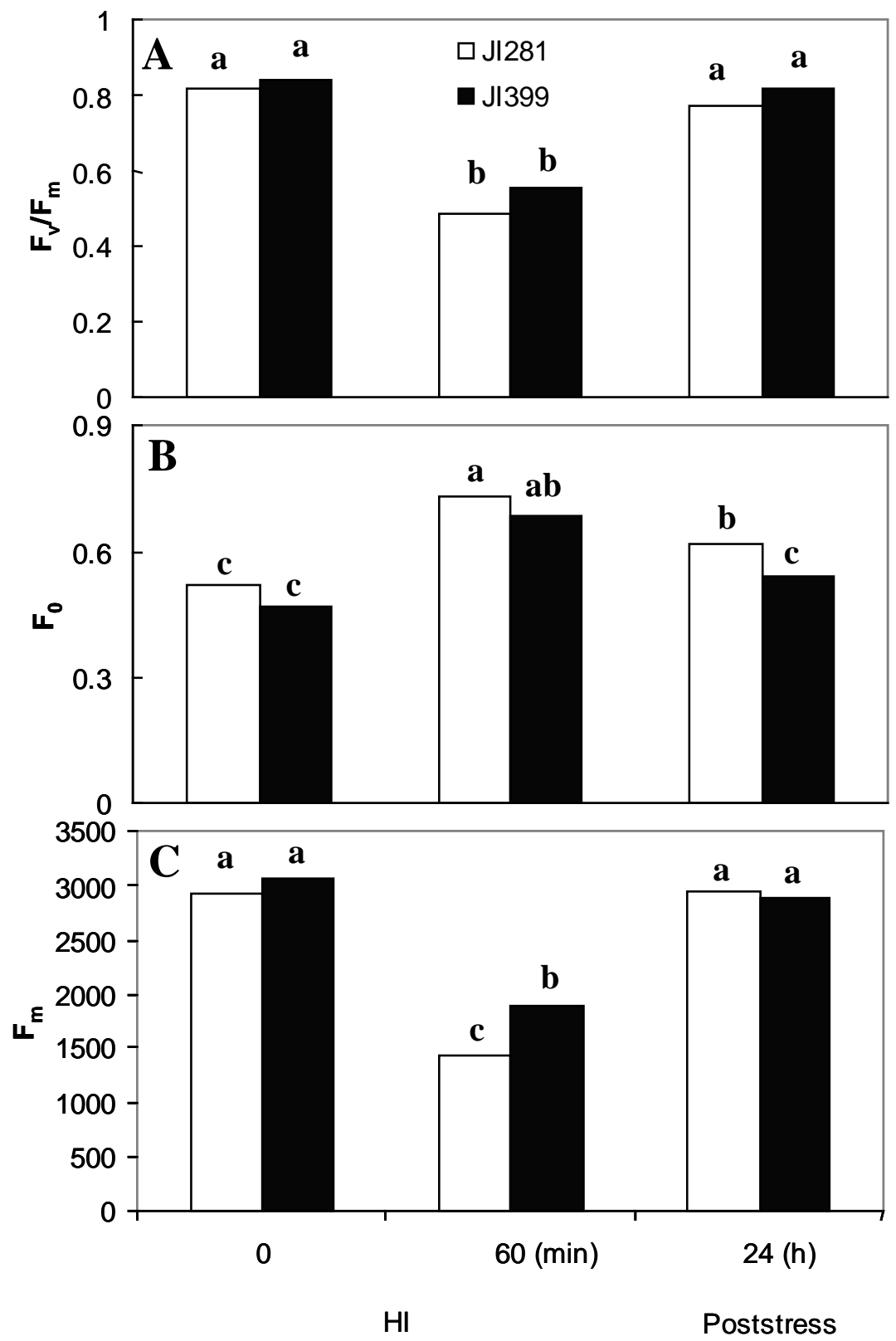

Fig 1.- Effect of HI on $F_{v} / F_{m}$ ratios $(A), F_{0}(B)$ and $F_{m}(C)$. The $F_{v} / F_{m}$ ratio, $F_{0}$ and $F_{m}$ were measured in three individual plants obtained from three independent experiments $(\mathrm{n}=9)$. The seedlings were grown at irradiance $200 \mu \mathrm{mol} \mathrm{m} \mathrm{m}^{-2}$. In the middle of the photoperiod, 3-week-old plants were exposed to HI (4000 $\left.\mu \mathrm{mol} \mathrm{m}^{-2} \mathrm{~s}^{-1}\right)$ for $60 \mathrm{~min}$ and returned to low irradiance. Means with different letters are significantly different according to Duncan's multiple range test $(\mathrm{P}=0.05)$. 


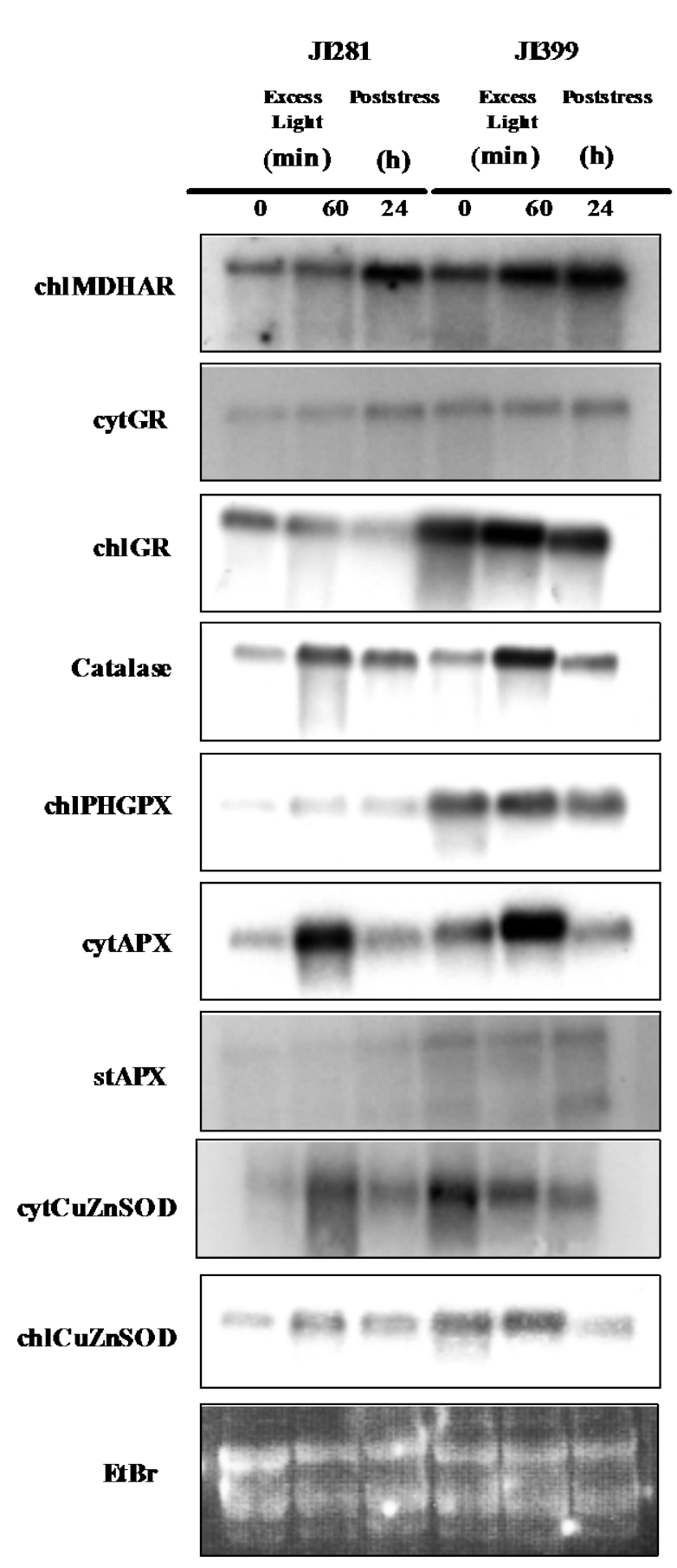

Fig 2.- Northern blot hybridisation analysis of poly (A+) RNA from pea plants exposed to HI. Names of probes used are indicated on the left. A representative Northern blot is shown. 